\section{Cureus

Received 06/03/2019

Review began 06/06/2019

Review ended 06/07/2019

Published 06/10/2019

\section{(C) Copyright 2019}

Naramala et al. This is an open access article distributed under the terms of the Creative Commons Attribution License CC-BY 3.0., which permits unrestricted use, distribution, and reproduction in any medium, provided the original author and source are credited.

\title{
Case Series of Cancer-associated Retinopathy (CAR)
}

Srikanth Naramala ${ }^{1}$, Jowairiyya Ahmad ${ }^{2}$, Sreedhar Adapa ${ }^{3}$, Frank Gavini ${ }^{4}$, Venu Madhav Konala 5, 6

1. Rheumatology, Adventist Medical Center, Hanford, USA 2. Rheumatology, Orange Regional Medical Center, Middletown, USA 3. Nephrology, The Nephrology Group, Visalia, USA 4. Cardiology, Adventist Medical Center, Hanford, USA 5. Hematology and Oncology, Ashland Bellefonte Cancer Center, Ashland, USA 6. Hematology and Oncology, King's Daughters Medical Center, Ashland, USA

Corresponding author: Srikanth Naramala,dr.srikanth83@gmail.com

\section{Abstract}

Cancer-associated retinopathy (CAR) is a rare paraneoplastic disorder of the retina leading to blindness, associated with multiple cancers. It can lead to rapid progressive visual deterioration with retinal pathology ranging from retinitis pigmentosa to retinal degeneration. It is caused by antibodies directed against retinal antigens. This uncommon syndrome is a remote effect, independent of the primary tumor or metastatic lesion. We describe two cases of CAR, as well as pathophysiology, clinical manifestation, diagnostic criteria, and treatment of cancerassociated retinopathy.

Categories: Ophthalmology, Oncology, Rheumatology

Keywords: retinopathy, cancer, paraneoplastic

\section{Introduction}

Cancer-associated retinopathy (CAR) is a rare paraneoplastic disorder of the retina. It is associated with multiple cancers. It can present with sudden, progressive loss of vision associated with photosensitivity. Other findings include ring scotoma, attenuated retinal arteriole, visual field defects, abnormal electroretinogram (ERG), and the presence of circulating serum autoantibodies specific to retinal antigens.

This uncommon syndrome is a remote effect, independent of the primary tumor or metastatic lesion. It is heterogeneous with a wide variety of different ocular symptoms.

\section{Case Presentation}

\section{Case 1}

A 39-year-old African American female presented to the clinic complaining of severe fatigue and increased urinary frequency. She had a past medical history of systemic lupus erythematosus - diagnosed based on arthritis, discoid rash, positive antinuclear antibody (ANA), ribonucleoprotein antibody (RNP), anti-Ro (SS-A) antibody, and controlled on hydroxychloroquine (Plaquenil). Further evaluation revealed a normal complete blood count (CBC) with differential, complete metabolic panel, erythrocyte sedimentation rate (ESR), Creactive protein (CRP), C3, C4, and double-stranded deoxyribonucleic acid (DNA). Urinalysis showed 2+ blood, along with red blood cells, and was negative for casts. She later underwent cystoscopy which revealed papillary urothelial carcinoma and subsequently underwent a left nephrectomy. Three years after her diagnosis of urothelial cancer, she presented with the 
sudden onset of visual blurring in both eyes. After an evaluation by a neuro-ophthalmologist, she was found to have constricted central and peripheral visual fields. Fluorescein angiography showed dense hyperfluorescence of the right eye and slight macular dystrophy, raising the concern for CAR. The genetic testing for mitochondrial disorders was negative. She had multiple positive anti-retinal antibodies which included anti-enolase, anti-GAPDH (glyceraldehyde 3-phosphate dehydrogenase), and anti-aldolase. Her treatment involved intravitreal triamcinolone injection in the right eye and she was concomitantly started on immunosuppressive therapy with prednisone and cyclosporine. There was no improvement in her vision with this therapy and it was subsequently switched to mycophenolate mofetil. She underwent a steroid implant six months later. Despite aggressive therapy, she had increased photosensitivity and was started on IVIG (intravenous immunoglobulin), complicated by hospitalization for aseptic meningitis. In the interim, she was under the care of urology and oncology for treatment of the bladder cancer with mitomycin.

\section{Case 2}

A 58-year-old African American female with a past medical history of hypertension, coronary artery disease, and diabetes mellitus type 2 presented with blurred vision. She described it as floaters and blackness in front of her eyes which later progressed to bilateral peripheral vision loss over a week's time. The ocular exam revealed extensive inflammatory findings in the anterior chamber, the posterior chamber, and the retinas bilaterally. She was placed on $1 \%$ prednisolone acetate eye drops and was referred to a retinal specialist for further evaluation of the abnormal retinal findings. The dilated fundoscopic exam revealed bilateral panuveitis, vitritis, retinal sheathing, and attenuation of vessels which was concerning for retinal vasculitis (Table 1). An autoimmune workup, including HLAB27, ANA, antineutrophil cytoplasmic antibody (ANCA), antiphospholipid antibody panel, serum angiotensin-converting enzyme (ACE), and rheumatoid factor (RF), was negative. An infectious disease workup, including human immunodeficiency virus (HIV), Lyme antibody titer, cytomegalovirus (CMV) antibodies, herpes simplex virus (HSV) antibodies, hepatitis serology, toxoplasmosis antibodies, Bartonella antibodies, syphilis rapid plasma reagin (RPR), and QuantiFERON-TB Gold Plus (Qiagen NV, Venlo, The Netherlands), were negative. Magnetic resonance imaging (MRI) of brain/orbits with contrast, as well as computed tomography (CT) of the chest, was non-revealing for significant pathology. She also had a history of chronic vaginal bleeding and back pain. Further examination revealed a pelvic mass, and a biopsy of the mass revealed endometrial carcinoma. This raised suspicion for CAR and the patient was tested for anti-retinal antibodies. She tested positive for anti-recoverin autoantibodies. She was initially treated with pulse dose steroids and referred for further management of her endometrial cancer. 


\section{Cureus}

\section{OCULUS DEXTER (RIGHT EYE)}

Intraocular pressure $14 \mathrm{~mm} \mathrm{Hg}$

Pupil equal and reacting to light

No afferent pupillary defect

Conjunctiva: white and quiet

Cornea: normal

Iris: normal

Anterior chamber: deep, $1+$ cell flare

Lens: trace nuclear cataracts

Vitreous: 2+ vitritis

Optic nerve: flat, sharp, good color

Macula: blunted

Retinal vessels: retinal sheathing, attenuated vessels $360^{\circ}$ from the posterior pole to the periphery

\section{OCULUS SINISTER (LEFT EYE)}

Intraocular pressure $16 \mathrm{~mm} \mathrm{Hg}$

Pupil equal and reacting to light

No afferent pupillary defect

Conjunctiva: white and quiet

\section{Cornea: normal}

Iris: normal

Anterior chamber: deep, $1+$ cell flare

Lens: trace nuclear cataracts

Vitreous: $2+$ vitritis

Optic nerve: flat, sharp, good color

Macula: blunted

Retinal vessels: retinal sheathing, attenuated vessels $360^{\circ}$ from the posterior pole to the periphery

\section{TABLE 1: Slit-lamp Examination}

\section{Discussion}

CAR is a rare paraneoplastic disorder of the retina leading to blindness and is associated with multiple cancers. It can lead to rapid progressive visual deterioration with retinal pathology ranging from retinitis pigmentosa to retinal degeneration. It is caused by antibodies directed against retinal antigens which include anti-recoverin, anti- $\alpha$-enolase, anti-carbonic anhydrase II, heat shock cognate protein 70 (HSC70), anti-transducin- $\alpha$ autoantibodies, and anti-GADPH found months to years before cancer detection [1]. It was first described in 1976 by Sawyer et al. as vision loss and photoreceptor dysfunction [2].

It is a subtype of paraneoplastic visual syndromes which consists of four entities: CAR, melanoma-associated retinopathy (MAR), paraneoplastic optic neuropathy (PON), and bilateral diffuse uveal melanocytic proliferation (BDUMP). In this review, we will focus on the pathophysiology, clinical manifestation, diagnostic criteria, and treatment of CAR.

Breast cancer, small-cell lung cancer, gynecological, and hematological malignancies are the most common tumors associated with CAR. Other less frequent cancers associated with CAR are hepatocellular carcinoma, thymoma, prostate cancer, and colon cancer. At least in $50 \%$ of the patients, the diagnosis of CAR precedes a cancer diagnosis (which also happened in our second patient) [3-5].

In the case series reported by Adamus involving 209 patients, women are more affected than men and the age group affected is 40 - 85 years [3]. Both cases described in our article are female patients. The onset of retinopathy from a cancer diagnosis can vary from weeks to 
months (lymphoma and lung cancer) to years (breast cancer and prostate cancer). The diagnosis may be preceded by the presence of anti-retinal antibodies [3].

Tumor antigens trigger an immune response which results in the development of autoantibodies that cross-react with a retinal protein that leads to retinal degeneration and cell death. The mechanism by which anti-retinal antibodies target retinal antigens causing photoreceptor damage is not entirely understood [6]. The postulated molecular mechanism is apoptotic death of photoreceptors mediated by caspase-dependent pathways, along with intracellular calcium influx [7-8].

The diagnosis of CAR is based on the signs and symptoms, as well as the diagnosis of systemic cancer. There are no standard diagnostic criteria for CAR. The diagnosis involves the detection of anti-retinal antibodies, electrophysiologic evidence of retinal degeneration (such as attenuated retinal vessels), degenerative and atrophic changes of the retinal pigment epithelium, and optic disc pallor with concurrent clinical manifestations [4, 9].

The clinical phenotype involves sudden, progressive, bilateral, painless visual deterioration with an acute, subacute, and rarely, chronic presentation. There is degeneration of the cones and rods with symptoms of photoaversion, prolonged glare after light exposure, reduced visual acuity, decreased color perception, and central scotoma. On ocular exam, the fundus usually appears normal; occasionally, some patients may show vascular attenuation, optic disc pallor, and retinal pigment abnormalities $[4,9]$.

ERG findings involve abnormal rods and cones. A multifocal and full-field electroretinogram is used, with distinct changes in the ERG. ERG is more sensitive than optical coherence tomography (OCT) which may only illustrate mild findings in early disease. Abnormalities of a and $b$ waves may be evident on ERG. Other findings on ERG include arterial attenuation, arteriolar sheathing, and periphlebitis. Optic disc pallor is seen in late cases. Slit-lamp examination rarely shows cellular debris in the anterior vitreous associated with low-grade inflammation in certain stages. Sawyer et al. showed there was photoreceptor degeneration of rods and cones with melanophages scattered in the outer retina and sparing of the ganglion cells in the inner retinal layer [2]. On OCT, diagnostic changes for pathologic anti-retinal antibodies include the loss of the outer retinal layer, such as the ellipsoid layer, and often show cystic spaces or occasionally mild schisis-like changes [7].

Greater than $50 \%$ of the patients with CAR will have serum autoantibodies that target retinal antigens. These antibodies have also been described in healthy individuals, although the appropriateness of the control sera in the study has been questioned $[3,7,10]$. In our patients, Case 1 was positive for anti-enolase, anti-GAPDH, and anti-aldolase antibodies. Case 2 was positive for anti-recoverin autoantibodies.

There are a variety of lab techniques to detect these antibodies which include immunohistochemistry (IHC), Western blot, and enzyme-linked immunosorbent assay (ELISA). By using the Western blot technique, the serum from a CAR patient reacts with recoverin. Recoverin is a $23-\mathrm{kDa}$ calcium-binding protein found on photoreceptors. It leads to the hypothesis that CAR is secondary to antibodies against tumor expressed recoverin that crossreacts with photoreceptors $[3,7,10]$.

The treatment of CAR involves treatment with corticosteroids associated with mild to moderate improvement in visual function based on anecdotal cases reports $[4,11]$. Given the pathophysiology of the autoimmune process, early institution of immunosuppressive therapy appears to improve the chance of treatment response. A review of the literature shows that patients had responses to IV immunoglobulin [12], alemtuzumab [13], rituximab [14], and 
plasmapheresis [15]. Plasmapheresis is hypothesized to remove anti-retinal antibodies, circulating immune complexes, and cytokines which are contributing to the immunological response. However, since plasmapheresis is performed in combination with steroids, immunosuppressive medications response cannot be attributed entirely to plasmapheresis itself. Most recently, a naturally-occurring immunomodulator, Tolpa Torf preparation (Torf Corp. Pharmaceuticals Ltd., Wrocławskie, Poland), was found to be effective in reducing antibody levels [16].

Despite the above treatments, the visual prognosis remains poor. Treatment of the underlying cancer is unlikely to affect any visual prognosis. Overall survival depends on the underlying tumor and staging at diagnosis, as well as treatment options available [17].

\section{Conclusions}

CAR is paraneoplastic retinopathy which might develop before cancer becomes clinically evident.

Screening for anti-retinal antibodies should be considered as part of the diagnostic workup in patients presenting with retinopathy with high pre-test probability. If paraneoplastic antiretinal antibodies are detected in the absence of a known cancer, workup for an occult neoplasm is appropriate.

There is no epidemiologic data available on CAR because it is infrequently encountered in clinical practice. There are also no guidelines for the treatment of CAR. Treatment mainly involves corticosteroids, immunosuppressive therapy, and IV immunoglobulin. Alemtuzumab, rituximab, and plasmapheresis can be considered in refractory cases.

\section{Additional Information \\ Disclosures}

Human subjects: Consent was obtained by all participants in this study. Conflicts of interest: In compliance with the ICMJE uniform disclosure form, all authors declare the following:

Payment/services info: All authors have declared that no financial support was received from any organization for the submitted work. Financial relationships: All authors have declared that they have no financial relationships at present or within the previous three years with any organizations that might have an interest in the submitted work. Other relationships: All authors have declared that there are no other relationships or activities that could appear to have influenced the submitted work.

\section{References}

1. Ohguro H, Yokoi Y, Ohguro I, et al.: Clinical and immunologic aspects of cancer-associated retinopathy. Am J Ophthalmol. 2004, 137:1117-19. 10.1016/j.ajo.2004.01.010

2. Sawyer RA, Selhorst JB, Zimmerman LE, Hoyt WF: Blindness caused by photoreceptor degeneration as a remote effect of cancer. Am J Ophthalmol. 1976, 81:606-13. 10.1016/00029394(76)90125-2

3. Adamus G: Autoantibody targets and their cancer relationship in the pathogenicity of paraneoplastic retinopathy. Autoimmun Rev. 2009, 8:410-14. 10.1016/j.autrev.2009.01.002

4. Chan JW: Paraneoplastic retinopathies and optic neuropathies . Surv Ophthalmol. 2003, 48:1238. 10.1016/s0039-6257(02)00416-2

5. Katsuta H, Okada M, Nakauchi T, Takahashi Y, Yamao S, Uchida S: Cancer-associated retinopathy associated with invasive thymoma. Am J Ophthalmol. 2002, 134:383-89. 10.1016/s0002-9394(02)01598-2

6. Heckenlively JR, Ferreyra HA: Autoimmune retinopathy: a review and summary. Semin 
Immunopathol. 2008, 30:127-34. 10.1007/s00281-008-0114-7

7. Grewal DS, Fishman GA, Jampol LM: Autoimmune retinopathy and antiretinal antibodies: a review. Retina. 2014, 34:827-45. 10.1097/IAE.0000000000000119

8. Shiraga S, Adamus G: Mechanism of CAR syndrome: anti-recoverin antibodies are the inducers of retinal cell apoptotic death via the caspase 9- and caspase 3-dependent pathway. J Neuroimmunol. 2002, 132:72-82. 10.1016/s0165-5728(02)00314-4

9. Jacobson DM, Thirkill CE, Tipping SJ: A clinical triad to diagnose paraneoplastic retinopathy . Ann Neurol. 1990, 28:162-67. 10.1002/ana.410280208

10. Adamus G, Ren G, Weleber RG: Autoantibodies against retinal proteins in paraneoplastic and autoimmune retinopathy. BMC Ophthalmol. 2004, 4:5. 10.1186/1471-2415-4-5

11. Keltner JL, Thirkill CE, Tyler NK, Roth AM: Management and monitoring of cancer-associated retinopathy. Arch Ophthalmol. 1992, 110:48-53. 10.1001/archopht.1992.01080130050025

12. Guy J, Aptsiauri N: Treatment of paraneoplastic visual loss with intravenous immunoglobulin: report of 3 cases. Arch Ophthalmol. 1999, 117:471-77. 10.1001/archopht.117.4.471

13. Espandar L, O'Brien S, Thirkill C, Lubecki LA, Esmaeli B: Successful treatment of cancerassociated retinopathy with alemtuzumab. J Neurooncol. 2007, 83:295-302. 10.1007/s11060006-9326-7

14. Mahdi N, Faia LJ, Goodwin J, Nussenblatt RB, Sen HN: A case of autoimmune retinopathy associated with thyroid carcinoma. Ocul Immunol Inflamm. 2010, 18:322-23. 10.3109/09273941003802379

15. Murphy MA, Thirkill CE, Hart WM Jr: Paraneoplastic retinopathy: a novel autoantibody reaction associated with small-cell lung carcinoma. J Neuroophthalmol. 1997, 17:77-83.

16. Adamus G, Amundson D, MacKay C, Gouras P: Long-term persistence of antirecoverin antibodies in endometrial cancer-associated retinopathy. Arch Ophthalmol. 1998, 116:251-53.

17. Goldstein SM, Syed NA, Milam AH, Maguire AM, Lawton TJ, Nichols CW: Cancer-associated retinopathy. Arch Ophthalmol. 1999, 117:1641-45. 10.1001/archopht.117.12.1641 